\title{
Prospectiva de la cobertura de la tierra en la Sub Cuenca del Río Mocal, Lempira, Honduras, Centroamérica.
}

Yessica Yamileth Sosa Reyes

\section{Resumen}

El objetivo de desarrollo de esta investigación se basó en el estudio de la dinámica de Cambio de la cobertura de la tierra en la Sub cuenca del Rio Mocal, generando para ello una Prospectiva por un Periodo de 20 años de la cobertura de la tierra. Definiéndose para ello la identificación de los problemas que inciden sobre el desarrollo territorial de la Sub cuenca, así mismo estableciendo escenarios de Destino (tendencial), escenarios de Porvenir y escenarios de Suceder. La metodología de implementación para este estudio se basó en la Identificación y caracterización de problemas, se desarrolló un diagnostico en donde se determinó las causas principales de los cambios de la cobertura de la Tierra y se formularon escenarios aplicados por la propuesta de metodología por Hevia (Araujo, O. R. 2005) para la Sub cuenca del Rio Mocal.Como resultado se obtuvieron periodos iniciales en donde se muestran el área de las coberturas de la tierra mostrando incrementos y decrementos del mismo, comenzando en el año 2014 y finalizando en el año 2035.

Palabras Clave: Escenarios, Cambios de Cobertura, Prospectiva.

\section{Abstract}

The target of development of this investigation based on the study of the dynamics of Change of the coverage of the ground in the Sub basin of the Rio Mocal, generating for it a Futurology for a Period of 20 years of the Coverage of the Earth. There being defined for it the identification of the problems that affect on the territorial development of the Sub basin, likewise establishing stages of (tendencial) Destination, stages of Future and stages of Happening. The implementation methodology for this study was based on the Identification and characterization of problems, there developed a 
diagnosis where one determined the prime movers of the changes of the Coverage of the Earth and there were formulated stages applied by the proposal of methodology by Hevia (Araujo, O. R. 2005) for the Sub basin of the Rio Mocal. As result there were obtained initial periods where they show themselves the area of the coverages of the ground showing increases and decreases of the same one, beginning in the year 2014 and finishing in the year 2035.

Key words: Stages, Changes of Coverage, Futurology.

Yessica Yamileth Sosa Reyes, (yessica.sosa04@gmail.com), Departamento de Ciencia y Tecnologías de la Información Geográfica. Facultad de Ciencias Espaciales, Universidad Nacional Autónoma de Honduras. 


\section{INTRODUCCIÓN}

Debido a los constantes cambios surgidos en el ambiente a lo largo de los años, por múltiples factores, climáticos, naturales, aumento de la población, incorporación de nuevas especies vegetales en coberturas distintas, ataque de plagas, etc, la cobertura de la tierra se ve afecta, distorsionando el origen de la naturaleza, con estos cambios la cobertura de la tierra se ve afectada.

Hoy en día las nuevas tecnologías de la información geográfica, han desarrollado herramientas de trabajo muy importantes, mediante las cuales se pueden llegar a conocer el comportamiento de aspectos físicos naturales de nuestro entorno y que de alguna manera permiten obtener resultados útiles para la disposición, manejo sostenible, la gestión de los recursos naturales, entre otros y que todos estos esfuerzos conlleven a la identificación de los cambios en la Cobertura de la tierra.

Uno de los afluentes más importantes del departamento de Lempira es la del Rio Mocal que tiene $56 \mathrm{Km}$. de largo, con un área de la cuenca de más de 1,000 km2 y una población de aproximadamente 100,000 habitantes. La gran mayoría de esa población es de origen lenca y sus municipios se encuentran entre los más pobres y menos desarrollado de Honduras según PNUD (PNUD, 2013).

Conocer la prospectiva de la Subcuenca del Rio Mocal, dará a conocer el destino 0 la tendencia, el porvenir y el suceder, con el fin de establecer alternativas para poder desarrollar dentro de la Sub cuenca, estrategias que contribuyan al mejoramiento de las condiciones de vida de las poblaciones, a que los usos del suelo sean los más acordes a las condiciones que en la Sub cuenca se encuentre, y a que las coberturas de la tierra sean los más indicados para el desarrollo socioeconómico de la Sub Cuenca.

El concepto de Prospectiva supone la fuerza creadora del hombre para dominar y transformar la naturaleza. En razón de ello está estrechamente relacionado con la noción de libertad, entendida como la capacidad que tiene la voluntad para obrar de una manera o de otra. Se podría decir que la construcción del futuro no fuera ni posible ni entendible si el hombre no tuviera la suficiente libertad para llevarla a cabo. Pero, a su vez, la voluntad obra movida por la claridad que le proporciona el intelecto (Mojica 1999).

El diseño de esta investigación se basó en un enfoque cuantitativo en donde se espera obtener visiones futuristas a 20 años, en cuanto lo tendencial y conservacionista. Utilizando estadísticas de las variables de cobertura de la tierra en la Sub cuenca del Rio Mocal, Lempira. Deductivo de acuerdo a las observaciones visuales en el 
terreno y observaciones visuales en las imágenes satelitales y probatorio de los diferentes tipos de coberturas de la tierra establecidos para esta investigación.

Es por ello que fue necesaria esta investigación, conocer de cierta manera como en una determinada zona, la dinámica del cambio que se había estado manifestando, puede dar indicios de como de acuerdo a la tendencia, el destino más adecuado 0 el porvenir puede llegar a marcar pautas para el control y manejo de los recursos naturales de la cobertura de la tierra y el uso adecuado del suelo.

\section{METODOLOGÍA}

Para realizar esta investigación la metodología se desarrolló en dos Fases:

\section{Fase l:}

Identificación y caracterización de problemas: se desarrolló un diagnostico en donde se determinó las causas principales de los cambios de la cobertura de la tierra (Físico, Socioeconómico y cultural), se identificaron algunos problemas relacionadas al cambio de la cobertura de la tierra de la sub cuenca del Rio Mocal.

\section{Fase Il:}

Formulación de Escenarios: Se aplicó la metodología propuestas por Hevia(Araujo, O. R. 2005) para el análisis prospectivo de la Subcuenta del Rio Mocal, que consistió en la siguiente descripción:

Destino: se desea el futuro por vía de descubrimiento. En este contexto corresponde a "adivinación", por ejemplo, el descubrimiento de la suerte de una persona, o de la "profecía" como aquella que trata acerca del destino de una población o de una cultura especifica.

Porvenir: futuro basado en la agrupación de posibles situaciones de la naturaleza en un plazo más o menos lejano. Descripción imaginaria, situándose en el contexto de lo utópico o de la ciencia-ficción.

Suceder: dado bajo un contexto histórico, el futuro se convierte en objeto del discurso de la acción.

La población y muestra definida para este estudio es: en el área de la Sub cuenca del Rio Mocal, y su muestra se basó en los porcentajes de cambio de la cobertura de la Tierra de la Sub cuenca del Rio Mocal. 


\section{RESULTADOS}

\section{Fase l: Identificación y Caracterización de Problemas}

Se Identificó el área de muestreo como la de la Sub cuenca del Rio Mocal, en el Sur del Departamento de Lempira, se desarrollaron talleres participativos en donde se elaboró un diagnósticopara la recolección de información básica de la caracterización de problemas en el tema ambiental de la Sub cuenca, así como de las condiciones físico naturales de la cobertura de la tierra, en esta etapa participaron miembros de diferentes municipios y organizaciones, así como también de instituciones gubernamentales y cooperantes internacionales, así como lo muestran las Figuras 1 y 2.

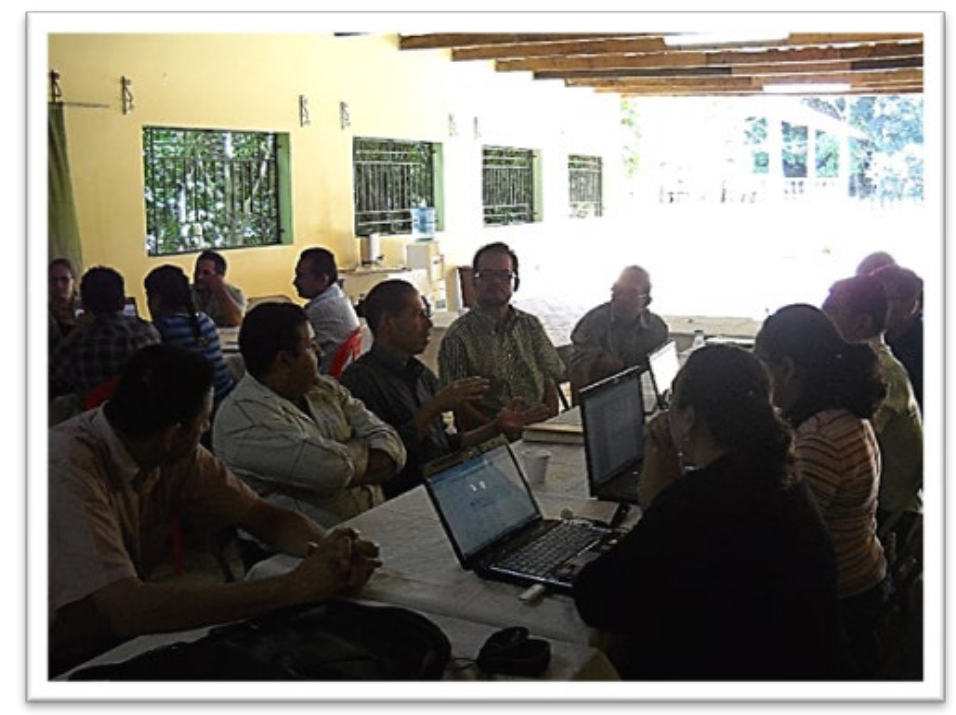

Figura 1. Diagnostico participativo para conocer problemas que afectan la Sub cuenca del Rio Mocal 


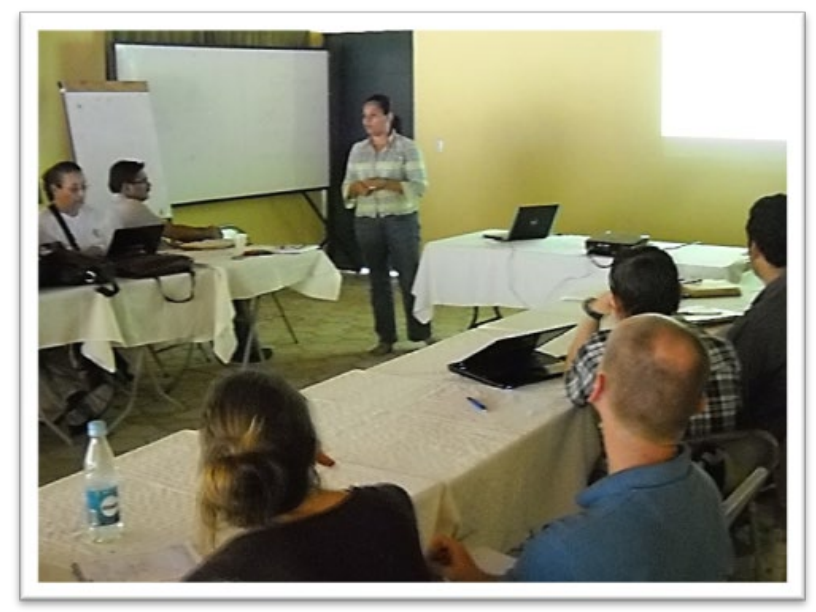

Figura 2. Diagnostico participativo con Cooperantes Internacionales

\section{Resultados de Diagnostico participativo}

Dentro de los resultados obtenidos para conocer la prospectiva a 20 años de la Sub cuenca del Rio Mocal, se desarrolló una caracterización de la Sub cuenca del Rio Mocal, así como también entrevistas a algunos ciudadanos de los municipios de la Sub cuenca del Rio Mocal.

\section{Caracterización de la Sub Cuenca del Rio Mocal}

\section{Ubicación Geográfica}

La Sub cuenca del Rio Mocal se encuentra ubicada geográficamente entre los Departamentos de Lempira y Ocotepeque, entre las coordenadas $14^{\circ} 33^{\prime} \mathrm{N}$ a 8850' 5,963"W tiene una extensión de 119,004.919 Ha., ubicada en el Occidente de Honduras, su extensión comprende $1119.00 \mathrm{M}^{2}$, los municipios del Departamento de Lempira son: Municipios de Gracias, San Manuel Colohete, Cololaca, San Sebastián, San Marcos de Caiquín, Tomalá, Tambla, San Andrés, Santa Cruz, Erandique, Valladolid, Gualcince, La Virtud, Mapulaca, Candelaria y Guarita, los municipios del Departamento de Ocotepeque: Belén Gualcho y San Marcos. (Ver Tabla 1) 
Tabla 1. Municipios de la Sub Cuenca del Rio Mocal

\begin{tabular}{|c|c|c|c|c|c|}
\hline No. & Departamento & Mnnicipio & $\begin{array}{l}\text { Area Total del } \\
\text { Municipio Fs. }\end{array}$ & Area Ka & Area Fa. \\
\hline $\mathbf{I}$ & \multirow[t]{16}{*}{ Lempira } & Gracias & 44904,912 & 6.8934 & 689,34 \\
\hline 2 & & Candelaria & 5319,797 & 4,0861 & 408,61 \\
\hline 3 & & Colalacx & 22535,442 & 3,05 & 305 \\
\hline 4 & & Erandiquu= & 29665,703 & 29,1751 & 2917,51 \\
\hline$\overline{5}$ & & Gualeince & 16277,859 & 71,4103 & 7141,07 \\
\hline$\overline{6}$ & & Guarita & 18034,071 & 2,6147 & 261,47 \\
\hline$\overline{7}$ & & L.a Virtud & 8896,458 & 68,2215 & 6822,15 \\
\hline$\overline{8}$ & & Mapulaca & 3248,905 & 12,8084 & 1280,84 \\
\hline$\overline{9}$ & & San Andró & 24682,833 & 245,7066 & 24570,66 \\
\hline 10 & & San Manual de Colohete & 18293,915 & 124,4768 & 12447,68 \\
\hline$\overline{11}$ & & San Sobzatian & 22195,15 & 206,1944 & 20619,44 \\
\hline 12 & & Santa Crux & 15134,625 & 96,5566 & 9655,66 \\
\hline 13 & & Tambla & 5856,84 & 43,6557 & 4365,57 \\
\hline 14 & & Tomalá & 4765,925 & 47,6598 & 4765,98 \\
\hline 15 & & Valladolid & 7830,983 & 63,3049 & 6330,49 \\
\hline 16 & & Sam Marces de Casiauin & 9657,988 & 62,1307 & 6213,07 \\
\hline 17 & \multirow[t]{2}{*}{ Ocotepeque } & Belén Gualcho & 15638,563 & 97,8633 & 9786,33 \\
\hline 18 & & San Marcos & 16922,235 & 4,2407 & 424,07 \\
\hline \multicolumn{3}{|c|}{ TOTAL } & 289862,204 & 1190,04 & 119004,94 \\
\hline
\end{tabular}

Fuente: Elaboración propia con datos SINIT

Límites de la Sub cuenca del Rio Mocal:

AL Norte: con los municipios del departamento de Lempira,

Al Sur: con el Rio Lempa y El Salvador,

Al Este: con el departamento de Intibucá y

Al Oeste: con el Departamento de Ocotepeque y el departamento de Lempira.

Dentro de la sub cuenca del Rio Mocal están comprendidos alrededor de 633 caseríos, de los cuales suman una población aproximada de 161,016 habitantes (INE, 2012).

La altitud máxima dentro de la Sub cuenca del Rio Mocal va desde los 2836 m.s.n.m., alturas mínimas que van desde los 56 m.s.n.m. y altura promedio es de 1,403.5 m.s.n.m. 


\section{Hidrografía}

El área de la Sub cuenca del Rio Mocal se encuentran alrededor de unas 280 redes de drenaje de ríos secundarios y quebradas superficiales, teniendo su nacimiento en el municipio de Belén Gualcho, Ocotepeque, con una longitud aproximada de 77 kilómetros.

La Sub cuenca del Rio Mocal se encuentra en la Región Lempa, número 14, que comprende los departamentos de; Intibucá, La Paz, Lempira y Ocotepeque (SEPLAN, 2011).

\section{Áreas Protegidas}

En el área de la Sub cuenca del Rio Mocal se ubican 3 áreas protegidas:

El Parque Nacional Montaña de Celaque el cual recientemente cambio de categoría y nombre a Reserva del Hombre y la Biosfera "Cacique Lempira, Señor de las Montañas", propuesto y aceptado en la Vigésimo Séptima Sesión del Consejo Internacional de Coordinación del Programa sobre el Hombre y la Biosfera (MABICC) de la UNESCO (Ambiente, 2015).

Esta área protegida se ubicada en el Departamento de Lempira en los municipios de Gracias, San Manuel de Colohete, Flores, en el Departamento de Ocotepeque en los municipios de BelenGualcho y Corquín.

Reserva Biológica Volcán Pacayita, ubicado en el Departamento de Lempira principalmente en los municipios de Cololaca y San Sebastián, en el Departamento de Ocotepeque los municipios de BelenGualcho y San Marcos.

Parque Nacional Congolón Piedra Parada y Coyocutena, ubicado geográficamente en el Departamento de Lempira en los municipios de San Andrés, Gualcince y Erandique.

\section{$\underline{\text { Cobertura Forestal }}$}

La cobertura Forestal de Honduras fue elaborada por la Unidad de Monitoreo Forestal del Instituto Nacional de Conservación y Desarrollo Forestal, Áreas Protegidas y Vida Silvestre (ICF) con el apoyo técnico y financiero del Programa Regional REDD/CCAD-GIZ durante los años 2013-2014 generado a partir de la clasificación e interpretación de imágenes del satélite Rapid Eye de los años 2012 y 2013. 
El Mapa cuenta con 5 macro categorías; Bosque, Agropecuario, Cuerpos de agua, Agroforestal y Otros usos (no bosque). Estas se dividen el 26 sub categorías.

Se ha estimado la cobertura forestal de nuestro país en 5,398.13 de hectáreas, distribuyéndose de la siguiente forma:

$57.5 \%$ de bosque Latífoliado $(3,101,574.31 \mathrm{ha})$

$36.3 \%$ de bosque de conífera $(1,960,511.08 \mathrm{ha})$

$5.3 \%$ de bosque mixto $(284,473.76 \mathrm{ha})$

$1.0 \%$ de bosque de mangle $(51,578.18 \mathrm{ha})$

La superficie cubierta de bosque representa el $48 \%$ del territorio hondureño.

De acuerdo a este mapa forestal, se encontraron un total de 14 clases de cobertura forestal en la sub cuenca del Rio Mocal (Ver Tabla 2).

Tabla 2. Coberturas Forestales en la Sub cuenca del Rio Mocal

\begin{tabular}{|l|l|l|l|}
\hline No. & Cobertura & Área Ha. & Porcentaje \\
\hline 1 & Bosque Latifoliado Húmedo & 2018,7 & 1,70 \\
\hline 2 & Bosque Latifoliado Deciduo & 5913,44 & 4,97 \\
\hline 3 & Bosque Mixto & 5807,11 & 4,88 \\
\hline 4 & Bosque de Conifera Denso & 18148,8 & 15,25 \\
\hline 5 & Bosque de Conifera Ralo & 3134,05 & 2,63 \\
\hline 6 & Vegetación Secundaria Húmeda & 6978,81 & 5,86 \\
\hline 7 & Vegetación Secundaria Decidua & 11391,1 & 9,57 \\
\hline 8 & Agricultura Tecnificada & 13,9875 & 0,01 \\
\hline 9 & Pastos / Cultivos & 59838,45 & 50,28 \\
\hline 10 & Cafetales & 2184,26 & 1,84 \\
\hline 11 & Otras superficies de Agua & 52,315 & 0,04 \\
\hline 12 & Zonas Urbanas Discontinuas & 171,203 & 0,14 \\
\hline 13 & Suelo Desnudo Continental & 178,28 & 0,15 \\
\hline 14 & Arboles dispersos fuera de Bosque & 3174,02 & 2,67 \\
\hline & & 119004,526 & 100 \\
\hline
\end{tabular}

Fuente: Mapa Forestal, 2014 
Los resultados fueron validados realizando un recorrido en campo, con áreas de muestreo de puntos al azar en todo el área, además de ello se realizaron pláticas con líderes municipales, ex alcaldes que mostraron interés en conocer cuánto y cómo el comportamiento de las coberturas de la tierra (ver Figura 3).

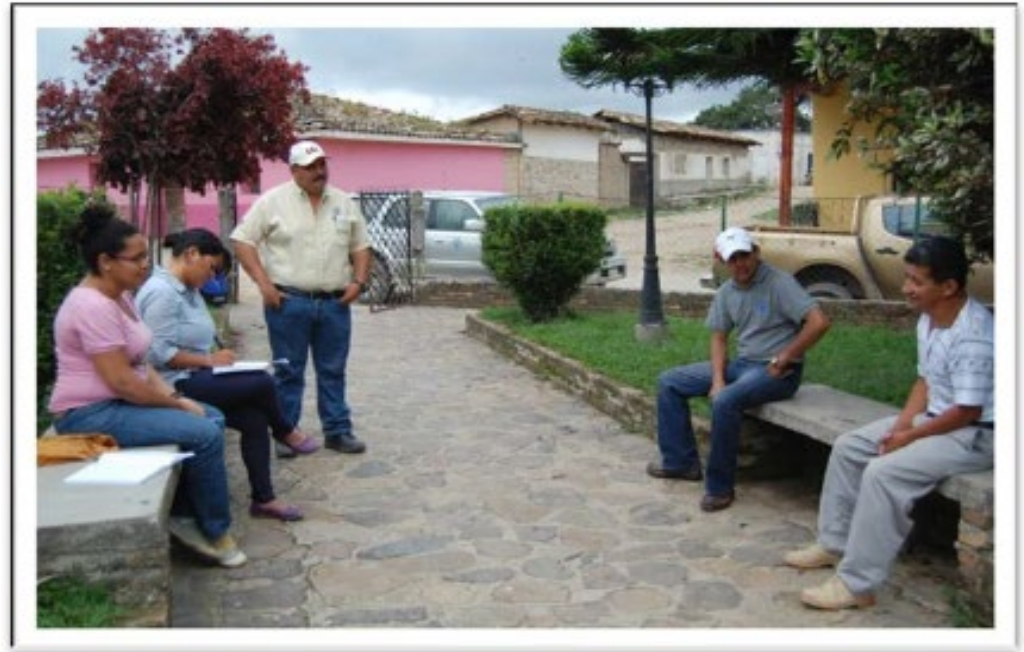

Figura 3. Reunión con autoridades del Municipio de San Sebastián, Lempira.

Se analizó entre todos los participantes sobre cuál es el área prioritaria para desarrollar el análisis del cambio de la cobertura de la tierra, lo cual se llegó a la conclusión sería la de toda la Sub cuenca del Rio Mocal que desemboca al Rio Lempa, por su interés ambiental, económico y social.

Dentro de los aspectos físico, socioeconómicos y ambientales se puede decir que existe un Modelo de Gestión de los Recursos Hídricos en el Sur de Lempira, en donde la gestión del agua en el sur del departamento de Lempira es realizada por el Comité Central Pro Agua y Desarrollo Integral de Lempira COCEPRADIL), que es una organización social de base integrada por diferentes juntas administradoras de agua, atendiendo 200 comunidades distribuidas en 16 municipios, con una cobertura de 10,000 familias conformadas por 40,540 personas (FAOHN, 2012).

Como resultado de la clasificación en el análisis multitemporal (Reyes, Y.Y. 2014) se desarrollóprevia a esta investigación se muestra la siguiente tabla conteniendo las áreas para las 6 coberturas de la sub cuenca del Rio Mocal.

En la Tabla 3, de la distribución de áreas de acuerdo a la cobertura de la tierra de la Sub cuenca del Rio Mocal por Hectárea y Porcentaje, el estudio basado en el 
análisis multitemporal de la Sub cuenca del Rio Mocal, en donde se tomaron como muestras a la variable de cobertura de la Tierra, los años 1988, 1998, 2000 y 2014.

Tabla 3. Tabla de distribución de Clases de Cobertura por año y en Porcentaje

\begin{tabular}{|l|l|l|l|l|l|l|l|l|}
\hline & Ha & $\%$ & Ha & $\%$ & Ha & $\%$ & Ha & $\%$ \\
\hline $\begin{array}{l}\text { Clases de } \\
\text { Cobertura }\end{array}$ & 1988 & & 1998 & & 2000 & & 2014 & \\
\hline Latifoliado & 8813.7 & 7,39 & 10788.3 & 9,05 & 10338.9 & 8,68 & 21730.4 & 18,25 \\
\hline Mixto & 42167.9 & 35,36 & 55111.3 & 46,25 & 69599.6 & 58,45 & 59678.1 & 50,11 \\
\hline Matorral & 54083.3 & 45,36 & 38721.8 & 32,49 & 30506.3 & 25,62 & 26324.9 & 22,11 \\
\hline Agricultura & 12916.9 & 10,83 & 14009.9 & 11,76 & 8552.16 & 7,18 & 11155.2 & 9,37 \\
\hline Nube & 726.66 & 0,61 & 534.24 & 0,45 & 81.63 & 0,07 & 196.2 & 0,16 \\
\hline $\begin{array}{l}\text { Area } \\
\text { Quemada }\end{array}$ & 528.39 & 0,44 & 0 & 0 & 0 & 0 & 0 & 0 \\
\hline TOTAL & 119236,85 & 100 & 119165,54 & 100 & 119078,59 & 100 & 119084,8 & 100 \\
\hline
\end{tabular}

Como se observa en la Figura 4, así como en laTabla 3, se muestra, que para la cobertura bosque de Latífoliado tiene un aumento en el año 2014 en comparación al año 1988, en el caso de la cobertura de Mixto tiene un aumento en el año 2000 a diferencia del año 1998 que presenta menos cobertura, con respecto a la cobertura de Matorral presenta mayor cobertura en el año 1988 en cambio para el año 2014 una disminución de su cobertura y para la cobertura de Agricultura los cambios son significativos teniendo un leve crecimiento en el año 1998, las coberturas de nube y área quemada no se presentan en todos los años por lo que sus valores son muy bajos. (Reyes, 2015).

Basándonos en estos resultados de datos en Hectáreas se proyectan los porcentajes de la cobertura de la tierra para los próximos 20 años se muestra en la figura 4.

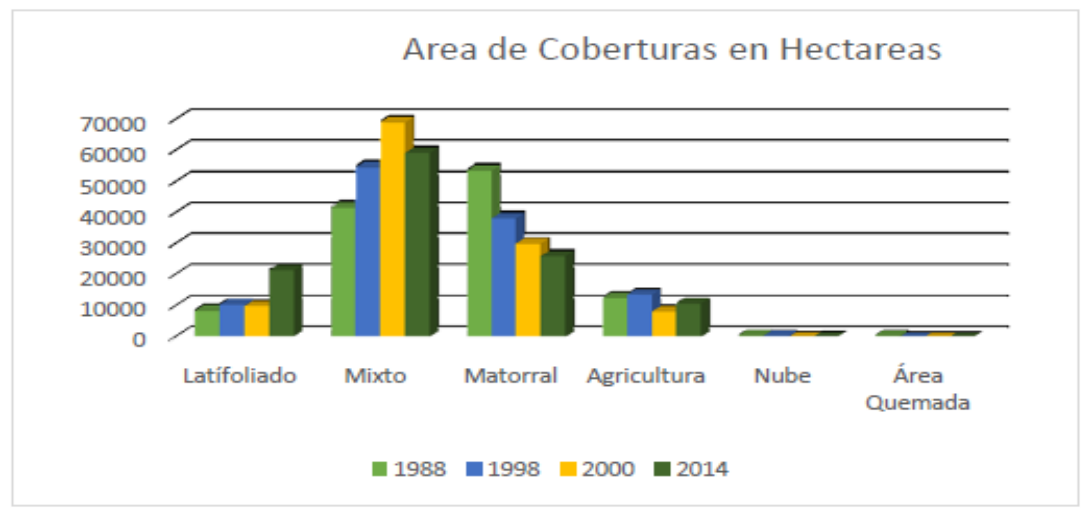

Figura 4. Tipos de coberturas en periodos de tiempo 
Muestra que para la cobertura de Bosque Mixto varia en los 4 años, mas sin embargo es la cobertura con más cambios de crecimiento, sin embargo la cobertura de Matorral varía de acuerdo al periodo de marcación, declinando sus valores encontrados.

\section{Fase II. Formulacion de Escenarios}

Para la formulación de los escenarios se muestra las variables de acuerdo a la representación de la cobertura de la tierra que se encontró en la Sub cuenca del Rio Mocal.

Durante la identificación de coberturas de la tierra en el muestreo realizado se encontró las siguientes:

\section{Bosque Latífoliado:}

En el Bosque Latífoliado de la Sub cuenca del Rio Mocal predominan las especies de hoja ancha, la condición siempre verde se refiere a que más del $75 \%$ de la cobertura está conformada por individuos que no pierden sus hojas. La altura de la cobertura de este tipo de bosques alcanza hasta los $40 \square 50 \mathrm{~m}$, en la zona del Mocal se observan como bosques densos, cerrados y dispersos, entre las principales especies que podemos encontrar en el área: Roble (Quercus sp), Encino (Quercus)., liquidámbar (Liquidambarstyraciflua), cedro (Cedrelaodorata), caoba (Swieteniamacrophylla), acacia (Delonix regia), ciprés (Cupressuslucitanica), cedro espino (Bombacopsisquinata), guancaste(Enterolobiumcyclocarpum), indio desnudo(Bursera simaruba), Cortes (Tabebuiachrysantha), ciruela silvestre, jobo etc., (ver Figura 5).
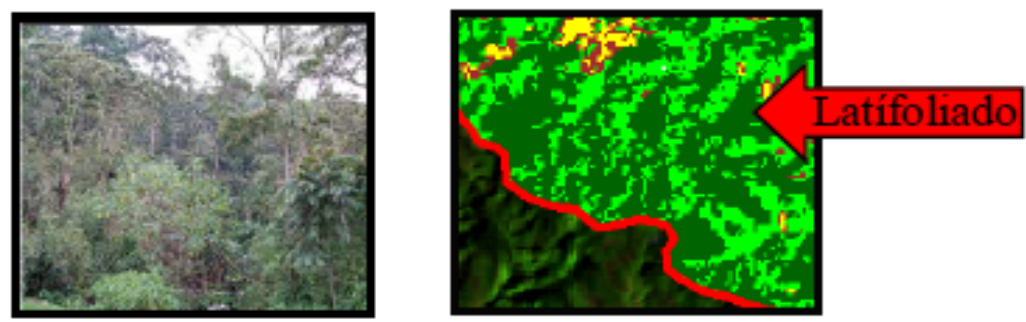

Figura 5. Ilustración de Bosque Latífoliado en Fotografía y en Imagen Satelital con proceso de clasificación y asignación de clase. 


\section{Bosque Mixto:}

Los bosques mixtos están asociados con las formaciones de bosque de latífoliado (Robles y Encinos) en combinación con coníferas. Las mezclas principales son de Pinusoocarpa y Pinusmaximinoi con varias especies del género Quercus, (ver Figura 6).
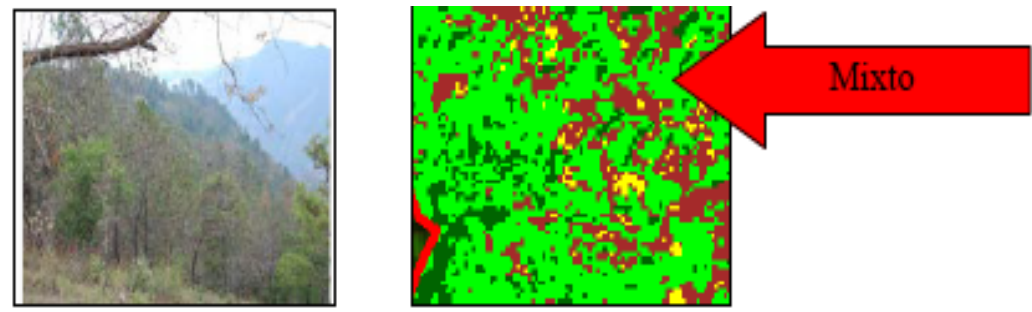

Figura 6. Ilustración de Bosque Mixto en Fotografía y en Imagen Satelital con proceso de clasificación y asignación de clase.

\section{Matorral:}

Es un campo caracterizado por una vegetación dominada por arbustos, plantas de porte herbáceo. El matorral también puede surgir como consecuencia de la actividad humana, (ver Figura 7).
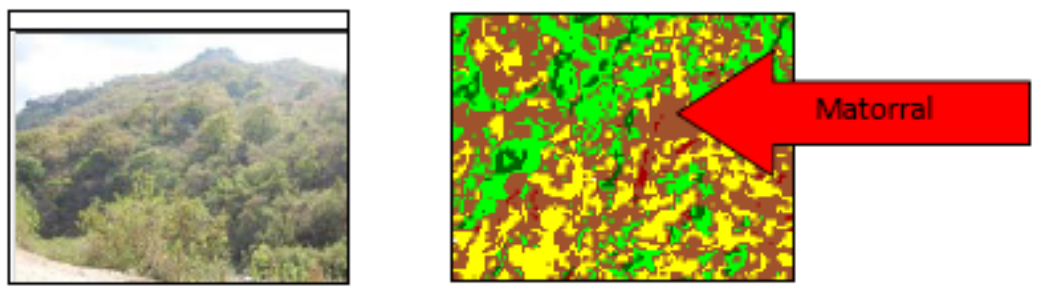

Figura 7. Ilustración de Matorral en Fotografía y en Imagen Satelital con proceso de clasificación y asignación de clase.

\section{Agricultura/ Pasto:}

La Agricultura: Es la actividad agraria que comprende todo un conjunto de acciones humanas que transforma el medio ambiente natural, con el fin de hacerlo más apto para el crecimiento de las siembras. Entre los principales cultivos agrícolas: maíz, frijol, hortalizas, caña de azúcar, plátanos, en condiciones dispersas. Muy poca presencia de agricultura tecnificada. 
El Pasto: Representa la superficie destinada a los diferentes cultivos agrícolas, áreas de pasto con o sin manejo y vegetación arbustiva, producto de la sucesión vegetal, al dejar abandonadas las áreas de cultivos, áreas destinadas a la actividad agrícola especialmente de la zona baja de la Sub cuenca del Rio Mocal, (ver Figura 8).
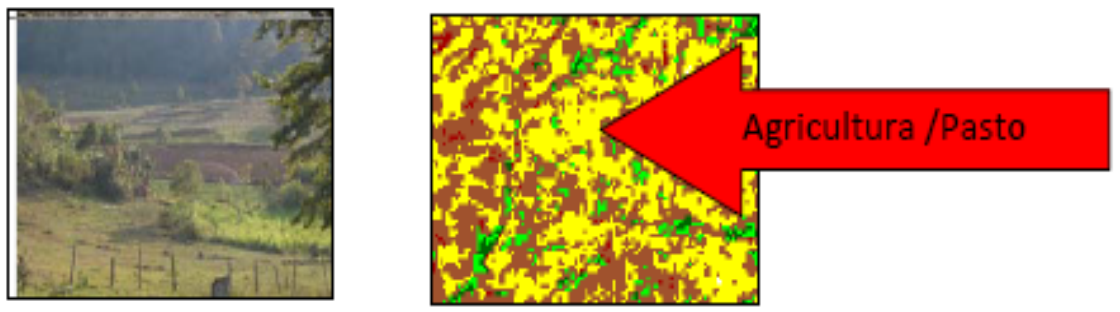

Figura 8. Ilustración de Agricultura/Pasto en Fotografía y en Imagen Satelital con proceso de clasificación y asignación de clase.

Con la utilización de se llevó a cabo con la utilización de herramienta de Matriz en donde hace una comparación de dos imágenes para conocer la ganancia y la perdida de cada una de ellas, esto con la finalidad sobre cómo se manifestó el comportamiento de crecimiento y decrecimiento de las coberturas y por ende la cobertura de la tierra para la Sub Cuenca del Rio Mocal.

Este proceso se realiza con la comparación de dos o más imágenes. Con el fin de conocer la dinámica del cambio ocurrido para este estudio desarrollando tres estados del tiempo, 1988-1998, 1998-2000 y 2000-2014.

Se generó una recodificación que automáticamente el sistema de Matriz lo realiza de acuerdo al número de clases encontradas, para este caso seis clases encontradas, 1: Nube, 2: Área Quemada, 3: Latífoliado, 4: Mixto, 5: Matorral y 6: Agricultura, (ver figura 9).

Los colores mostrados en la Figura 10 muestran las áreas con cambio, sin cambio y las coberturas que se mantenían, a continuación, la descripción de la asignación de los colores. 


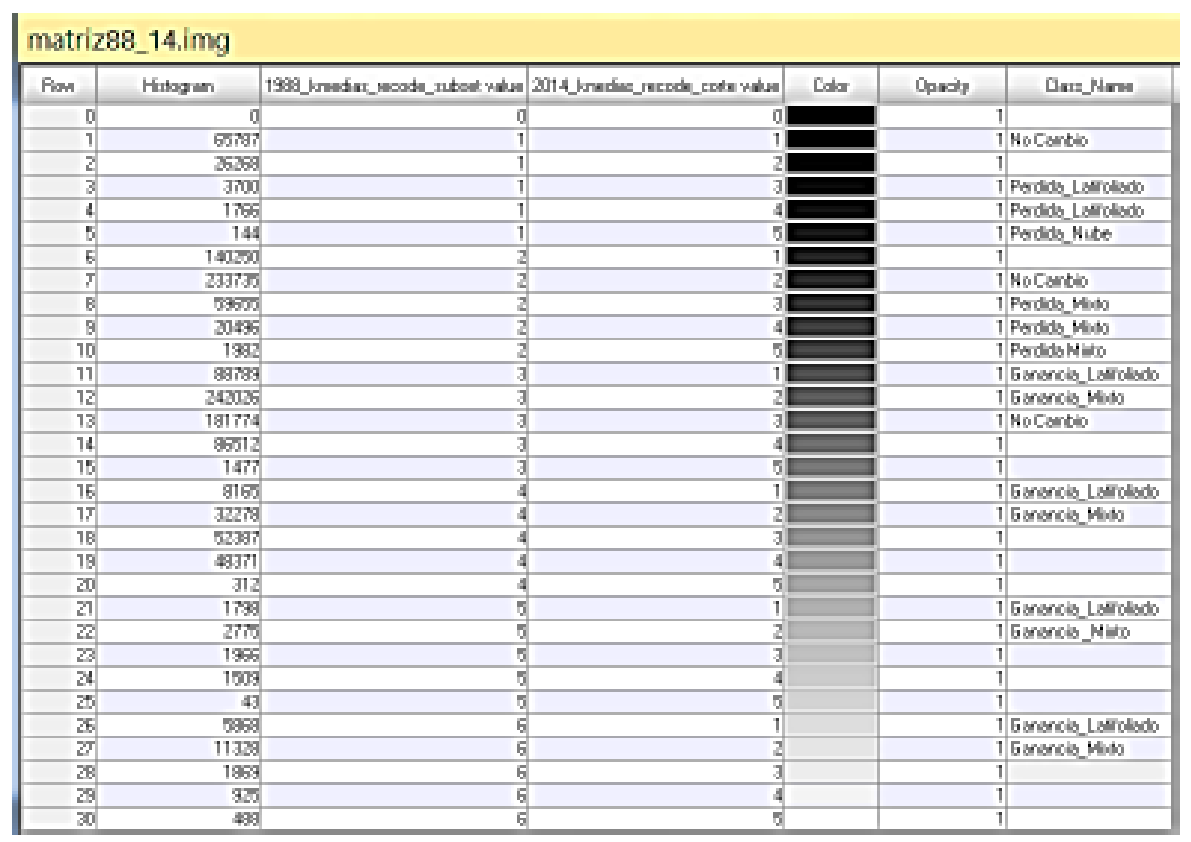

Figura 9. Matriz para la Validación de la Información de la cobertura de la tierra

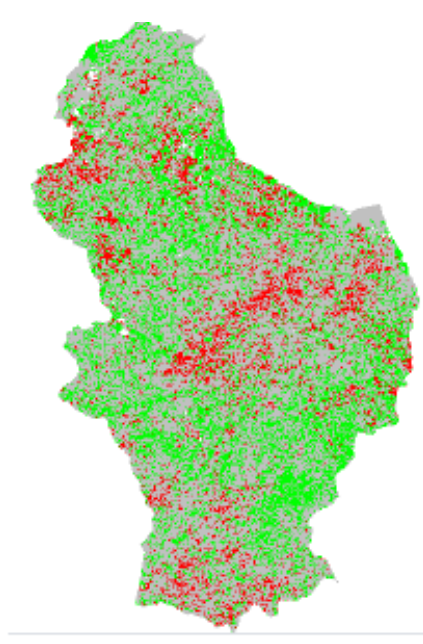

Periodo 1988-1998
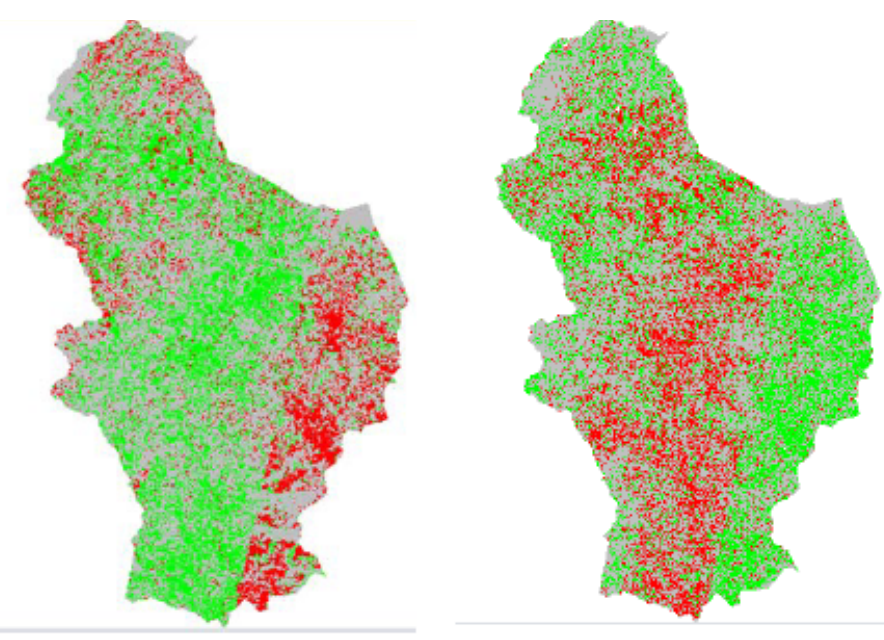

Periodo 1998-2000

Periodo 2000-2014

Figura 10. Decremento y Aumento de las coberturas de la Sub cuenca del Rio Mocal. 


\section{Resultado de la Matriz de cada periodo}

- Rojo: o decremento, cuando ha ocurrido cambios en la cobertura de Latífoliado a Mixto, matorral a pasto 0 agricultura, de Latífoliado a matorral, coberturas de mixto a matorral.

- Verde: 0 aumento de la cobertura cuando cambia de mixto a Latífoliado, de Matorral a Mixto, Matorral a Latífoliado.

- Gris: son aquellas áreas que se mantienen sin cambio en las imágenes.

En base a este análisis podremos definir que para el escenario de Destino estos serán los valores de la cobertura de la tierra de la Sub cuenca del Rio Mocal.

\section{ESCENARIO DE DESTINO:}

De acuerdo a su proceso de análisis en donde se desea se descubra el futuro de un estado de acuerdo a lo que por naturaleza se ha ido desarrollando el entorno, en la sub cuenca del Rio Mocal el Escenario Destino se construyó a partir de indicadores de monitoreo ambiental, como ser; planes de manejo, áreas bajo protección contra incendios forestales, Ríos principales abastecedores de agua, entre otros, así mismo los socioculturales como ser aquellos relacionados con la organizaciones que velan por el funcionamiento sostenible y efectivos de sus áreas de producción, poblaciones asentadas en áreas de uso especial, organizaciones involucradas en el mejoramiento productivo, organismo locales involucrados en los procesos ambientales, etc.

En este escenario se puede apreciar que de acuerdo a lo ya antes mencionado sobre los diferentes tipos de cobertura de la Tierra encontrados se muestra un posible Escenario de Destino (Ver Figura 11). 


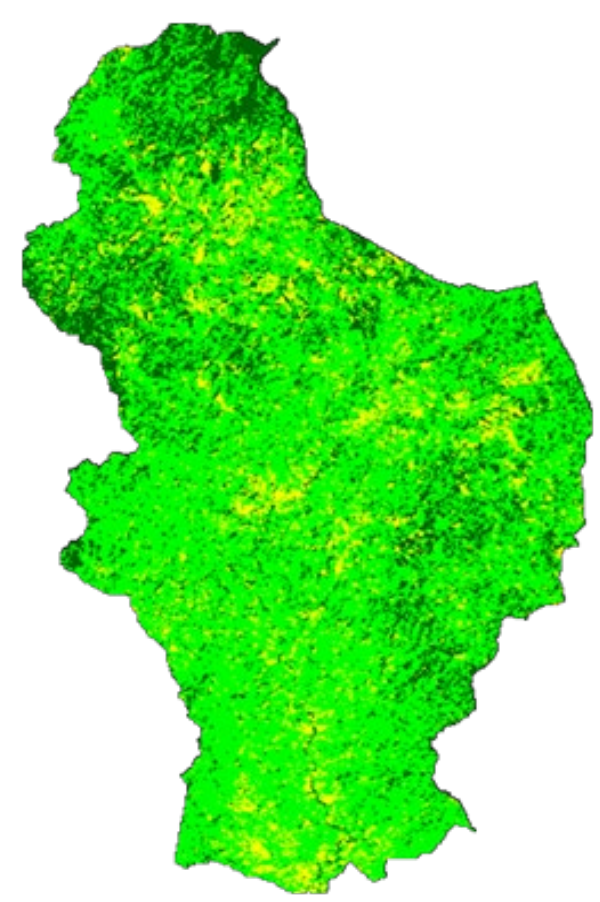

Figura 11. Escenario Destino

Tabla 4. Matriz para la validación de la información de la cobertura de la Tierra.

\begin{tabular}{|l|l|l|l|l|}
\hline Clase de Cobertura & $2014 \mathrm{Ha}$ & $\%$ & $2035 \mathrm{Ha}$ & $\%$ \\
\hline Latifoliado & 21730.4 & 18,25 & 26715,38 & 22,4 \\
\hline Mixto & 59678.1 & 50,11 & 57906,15 & 48,6 \\
\hline Matorral & 26324.9 & 22,11 & 25249,92 & 21,2 \\
\hline Agricultura & 11155.2 & 9,37 & 9213,35 & 7,73 \\
\hline TOTAL & 119084,8 & 100 & 119084,8 & 100 \\
\hline
\end{tabular}

Como lo muestra la Tabla 4, los valores de acuerdo al año inicial 2014 y en hectáreas, así como el año final 2035 y en hectáreas de las coberturas de la tierra de la Sub cuenca del Rio Mocal, mostrando el posible escenario destino para la cobertura de bosque latífoliado sufre un aumento en la cobertura pasando de un 18, 25\% a un 22, $4 \%$ un aumento de $4.15 \%$, así mismo el valor de la cobertura de Mixto sufre una leve disminución en su cobertura del $1.51 \%$. 


\section{ESCENARIO DE PORVENIR:}

Para desarrollar este escenario fue necesaria la utilización de matriz de confusión en donde se obtuvieron las tasas de cambio de la cobertura de la tierra, previsto en el análisis multitemporal de la cobertura de la tierra de la sub cuenca del Rio Mocal (Reyes, 2015), en este escenario muestra como la cobertura de Bosque Latífoliado se ve mayor mente influenciado, con remarcada visualización en la cobertura de matorral, dejando entrever pequeñas manchas de la cobertura agrícola, (ver Figura 12).

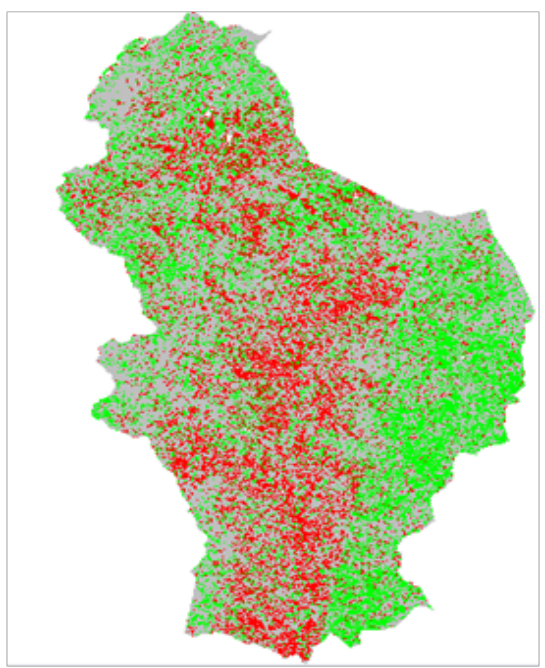

Figura 12. Tasas de Cambio en la cobertura de la tierra

Tabla 5. Tasa de cambio en la cobertura de la Tierra de la Sub cuenca del Rio Mocal.

\begin{tabular}{|c|c|c|c|}
\hline Cobertura & $\begin{array}{l}\text { Superficie Has. } \\
2000\end{array}$ & $\begin{array}{l}\text { Superficie Has. } \\
2014\end{array}$ & $\begin{array}{l}\text { Tasa de } \\
\text { Cambio }\end{array}$ \\
\hline Latifoliado & 10338.9 & 21730.4 & +5.41 \\
\hline Mixto & 69599.6 & 59678.1 & -1.09 \\
\hline Matorral & 30506.3 & 26324.9 & -1.04 \\
\hline $\begin{array}{l}\text { Agricultura } \\
\text { Pasto }\end{array}$ & 8552.16 & 11155.2 & +1.90 \\
\hline Desconocido & & 11351.4 & \\
\hline
\end{tabular}

La Tabla 5, muestra cambios positivos en la recuperación de la Cobertura de Latífoliado con +5.41 , al igual que la Agricultura / Pasto, las coberturas de Mixto y Matorral muestran una leve perdida con respecto al periodo anterior. Sin embargo 
en la cobertura de Bosque Mixto y Matorral se muestra un dato negativo debido a la dominancia de la especie, este dato significativo resalta la secuencia con que se ha venido desarrollando el crecimiento de cada una de las coberturas, influenciada por varios factores.

De acuerdo a este resultado el Escenario Porvenir a 20 años de la Sub cuenca del Rio Mocal se manifiesta en donde la cobertura de Bosque Latífoliado se ve mayor mente influenciada y muy similar a la cobertura de Bosque Mixto, teniendo pequeñas visualizaciones de la cobertura de agricultura y pasto, (ver Figura 13).

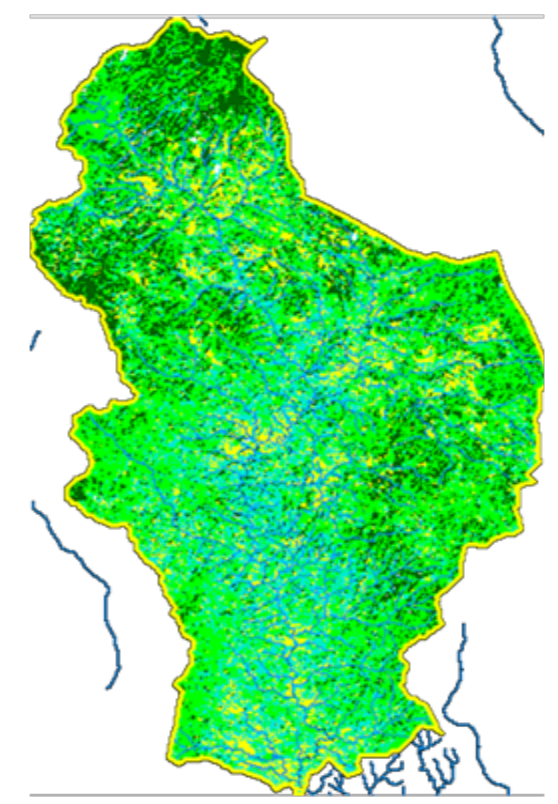

Figura 13. Escenario Porvenir, sub cuenca Rio Mocal

Tabla 6. Tipo de Cobertura para escenario Porvenir, sub cuenca del Rio Mocal.

\begin{tabular}{|l|l|l|l|l|}
\hline Tipo de Cobertura & $2014 \mathrm{Ha}$ & $\%$ & $2035 \mathrm{Ha}$ & $\%$ \\
\hline Latifoliado & 21730.4 & 18,25 & 37165,38 & 31.2 \\
\hline Mixto & 59678.1 & 50,11 & 67906,15 & 57.02 \\
\hline Matorral & 26324.9 & 22,11 & 949,92 & 0.79 \\
\hline Agricultura & 11155.2 & 9,37 & 1713,35 & 1.44 \\
\hline Desconocido & - & - & 11350 & 9.55 \\
\hline TOTAL & 119084,8 & 100 & 119084,8 & 100 \\
\hline
\end{tabular}


La Tabla 6, muestra los valores de acuerdo al año inicial 2014 y en hectáreas, así como el año final 2035 y en hectáreas de las coberturas de la tierra de la Sub cuenca del Rio Mocal, de acuerdo al Escenario de Destino se muestra que para lacobertura de latífoliado sufre un aumento pasando de un $18,25 \%$ a un $31,2 \%$ un aumento de $12,95 \%$, así mismo el valor de la cobertura de Mixto sufre un aumento en su cobertura del $6.91 \%$, en cambio la cobertura de matorral cambia la tendencia pasando estos valores a formar parte de la cobertura de Latífoliado y la cobertura de boque mixto, ocasionando cambios altos en la cobertura de la tierra, así mismo se puede apreciar el cambio en la agricultura.

\section{ESCENARIO DE SUCEDER:}

El resultado de este escenario se desarrolló tomando en cuenta los factores que históricamente se manifiestan en el subcuenca del rio Mocal, para esta situación se tomó de base el análisis antes realizado en donde a través de un análisis multitemporal de un periodo de 23 años, en donde se conoció cuál es la interacción de la dinámica del crecimiento de acuerdo a los factores climáticos, ambientales y productivos de la sub cuenca del rio Mocal. Aquí se encontró que la cobertura de bosque latífoliado es la que más predomina, teniendo a la cobertura de bosque mixto con pequeñas áreas, la cobertura de matorral se manifiesta de manera esporádica en comparación a la cobertura de agricultura y pasto. (Ver Figura 14).

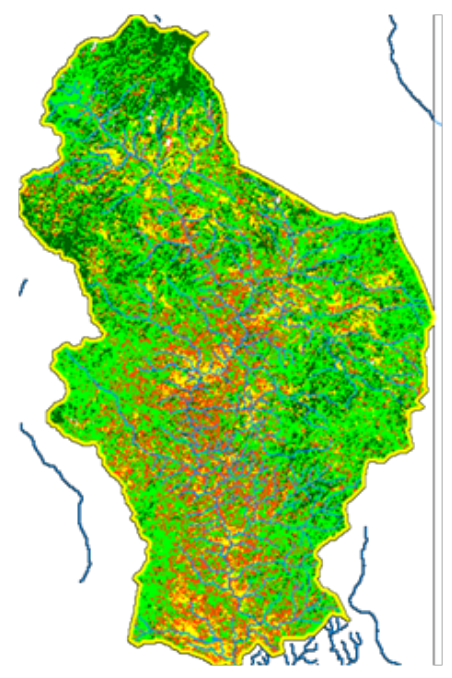

Figura 14. Cobertura de la tierra en Escenario Suceder. 
Tabla 7. Tipo de Cobertura para escenario Suceder, sub cuenca del Rio Mocal.

\begin{tabular}{|l|l|l|l|l|}
\hline Tipo de Cobertura & $2014 \mathrm{Ha}$ & $\%$ & $2035 \mathrm{Ha}$ & $\%$ \\
\hline Latifoliado & 21730.4 & 18,25 & 26715,38 & 22,4 \\
\hline Mixto & 59678.1 & 50,11 & 57906,15 & 48,6 \\
\hline Matorral & 26324.9 & 22,11 & 7813,34 & 6.56 \\
\hline Pasto & 0 & 0 & 17436,58 & 14.71 \\
\hline Agricultura & 11155.2 & 9,37 & 9213,35 & 7,73 \\
\hline TOTAL & 119084,8 & 100 & 119084,8 & 100 \\
\hline
\end{tabular}

La Tabla 7, muestra los valores de acuerdo al año inicial 2014 y en hectáreas, así como el año final 2035 y en hectáreas de las coberturas de la tierra de la Sub cuenca del Rio Mocal, mostrando de acuerdo al Escenario de Suceder se muestra que para la Cobertura de Latífoliado se mantiene, al igual que la cobertura de Mixto, y la de agricultura, en cambio la cobertura de matorral se fracciona generando 0 siendo más visible el cambio en la cobertura de la tierra a una cobertura de pasto, de acuerdo a la tendencia y a las necesidades básicas de la sub cuenca del Rio Mocal, obteniendo para la cobertura de matorral un porcentaje de $6.56 \%$ mientras que el pasto con un porcentaje de $14.71 \%$.

\section{DISCUSIÓN Y CONCLUSIONES}

- Como apoyo a la prospectiva Sub cuenca del Rio Mocal utilizando las variables de cobertura de la tierra en base estudios anteriormente desarrollados para esta investigación con el mismo propósito, continuar con la misma investigación en donde me muestra resultado en donde se puede proporcionar para mejorar o alertar sobre los diferentes escenarios que se muestran en la Sub cuenca del Rio Mocal.

- En cuanto a la cobertura de matorral muestra una disminución del $15.5 \%$ en el Escenario Suceder comenzando con un $22.11 \%$ lo que establece que esta cobertura tubo un cambio significativo con posibilidades de pasar de una cobertura a otra, o dejando invadir de acuerdo a las necesidades de la población. el alto descenso.

- Tomando en cuenta la tendencia al crecimiento de la cobertura de la tierra que se le da a cada uno de ellos podemos determinar que no son del todo confiables, influirán otros factores como los climáticos, llámese Huracanes, Fenómeno del Niño o Niña en donde son condiciones naturales difícilmente de 
manipular, pero en donde sí se pueden evitar o mantener alternativas ante los posibles cambios.

- Una de los propósitos de desarrollar análisis de prospectiva es dar a conocer cuáles son los posible enfoques, escenarios o visiones que se tiene con respecto a una área geográfica, en este caso conociendo la situación de extrema pobreza que existe en el corredor en donde se ubica la sub cuenca del rio Mocal, muestra la gran necesidad de establecer a futuro alternativas que ayuden a minimizar estos aspectos.

\section{BIBLIOGRAFÍA}

- Ambiente, M. (2015). Mi Ambiente. Obtenido de http://www.miambiente.gob. $\mathrm{hn} /$ index.php/noticias/48-consejo-internacional-inscribio-el-parque-nacionalmontana-de-celaque-como-reserva-del-hombre-y-la-biosfera-cacique-lempirasenor-de-las-montanas

- Araujo, O. R. (2005). Metodología de Escenarios: ¿Utopía o Concreción Prospectiva en las Ciencias Sociales? Obtenido de en http://www.iaeal.usb. ve/90/90-3.pdf

- FAO, F. a. (2005). Sistema de Clasificación de cobertura de la Tierra, conceptos de clasificación y Manual del Usuario versión 2 del programa. Roma: FAO.

- FAOHN. (2012). Taller de Búsqueda de consenso para la Formulación de Estrategia de Bienes y Servicios Ambientales (BSA) de los Municipios Verdes del Departamento de Lempira. Tegucigalpa, MDC, Honduras.

- INE, I. N. (2012). Censo Población y Vivienda. Tegucigalpa, Honduras.

- PNUD, (2013), Informe de Índice de Desarrollo Humano Honduras 2013

- REDDICCAD $₫ G I Z$, P. (2011). Tipos de Bosques y contexto del mapeo de la cobertura forestal. Obtenido de http://www.reddccadgiz.org/documentos/ doc_1170376601.pdf

- Reyes, Y. Y. (2015). AnálisisMultitemporal de Cobertura de la Tierra de la Subcuenca del Rio Mocal, Lempira, Honduras, durante el periodo de los años 1988 al 2014.

- SEPLAN, S. T. (24 de septiembre de 2011). SINIT CD de Presentación. Tegucigalpa, Honduras. 